\title{
Revenue Generation for Truthful Spectrum Auction in Dynamic Spectrum Access
}

\author{
Juncheng Jia ${ }^{\dagger} \quad$ Qian Zhang ${ }^{\dagger}$ \\ ${ }^{\dagger}$ Computer Science and Engineering \\ Hong Kong University of Science and \\ Technology, CHINA \\ \{jiajc, qianzh, qinzhang\}@cse.ust.hk
}

\author{
Qin Zhang ${ }^{\dagger} \quad$ Mingyan Lius \\ ${ }^{\S}$ Electrical Engineering and Computer Science \\ University of Michigan \\ USA \\ mingyan@eecs.umich.edu
}

\begin{abstract}
Spectrum is a critical yet scarce resource and it has been shown that dynamic spectrum access can significantly improve spectrum utilization. To achieve this, it is important to incentivize the primary license holders to open up their under-utilized spectrum for sharing. In this paper we present a secondary spectrum market where a primary license holder can sell access to its unused or under-used spectrum resources in the form of certain fine-grained spectrumspace-time unit. Secondary wireless service providers can purchase such contracts to deploy new service, enhance their existing service, or deploy ad hoc service to meet flash crowds demand. Within the context of this market, we investigate how to use auction mechanisms to allocate and price spectrum resources so that the primary license holder's revenue is maximized. We begin by classifying a number of alternative auction formats in terms of spectrum demand. We then study a specific auction format where secondary wireless service providers have demands for fixed locations (cells). We propose an optimal auction based on the concept of virtual valuation. Assuming the knowledge of valuation distributions, the optimal auction uses the Vickrey-Clarke-Groves (VCG) mechanism to maximize the expected revenue while enforcing truthfulness. To reduce the computational complexity, we further design a truthful suboptimal auction with polynomial time complexity. It uses a monotone allocation and critical value payment to enforce truthfulness. Simulation results show that this suboptimal auction can generate stable expected revenue.
\end{abstract}

\section{Categories and Subject Descriptors}

C.2.1 [Computer Systems Organization]: Computer Communication Networks

\section{General Terms}

Algorithm, Design, Economics

Permission to make digital or hard copies of all or part of this work for personal or classroom use is granted without fee provided that copies are not made or distributed for profit or commercial advantage and that copies bear this notice and the full citation on the first page. To copy otherwise, to republish, to post on servers or to redistribute to lists, requires prior specific permission and/or a fee.

MobiHoc'09, May 18-21, 2009, New Orleans, Louisiana, USA.

Copyright 2009 ACM 978-1-60558-531-4/09/05 ...\$5.00.

\section{Keywords}

Spectrum Auctions, Mechanism Design

\section{INTRODUCTION}

Radio spectrum is a critical yet scarce resource in the provision of wireless telecommunications services. Acquisition of a spectrum license is the very first, and also one of the most difficult and costly procedures in the development and deployment of any new wireless service as well as its market entry. Because of the externality in spectrum usage such as electromagnetic interference, the allocation of spectrum resources has been strictly coordinated and regulated.

Traditionally, spectrum allocation has been done in a centralized, static, and wholesale fashion, where long-term (on the order of 10s of years) spectrum leases/contracts are sold covering very large geographical regions (see for instance the regional spectrum allocation in the recent FCC's $700 \mathrm{MHz}$ auction, and the $27 \mathrm{GHz}$ allocation in Australia). As a result, the type of buyers who can meaningfully participate in this type of allocation is limited, due to the large amount of capitals needed.

Under this type of static allocation, there is increasing evidence that spectrum resources are not being efficiently utilized [1], and that there exists abundant spectrum opportunity currently unexploited within licensed bands. At the same time, wireless devices are enjoying ever greater capability to detect spectrum availability and flexibility to adjust operating frequencies [2]. These observations have led to a push for the original license holders and operators, a primary example being TV broadcasters ${ }^{1}$, to open up access to secondary unlicensed users [3], so as to improve spectrum utilization, a concept known as dynamic spectrum access [4]. With dynamic spectrum access, secondary unlicensed users may take advantage of (instantaneous) spectrum opportunities that exist in spectrum owned by a primary user. This has motivated a large number of recent studies on how a secondary user should perform opportunistic spectrum access and how primary and secondary users could co-exist $[5,6]{ }^{2}$

There are several unique challenges in such a new spectrum sharing paradigm. Firstly, we believe that unless there

\footnotetext{
${ }^{1}$ Indeed the FCC on November 4, 2008 approved unlicensed wireless devices that operate in the empty white space between TV channels, after four years of effort.

${ }^{2}$ In this paper, we use the terms primary license holder, primary user and sellers interchangeably, while we use the terms secondary wireless service providers, secondary users and buyers interchangeably.
} 


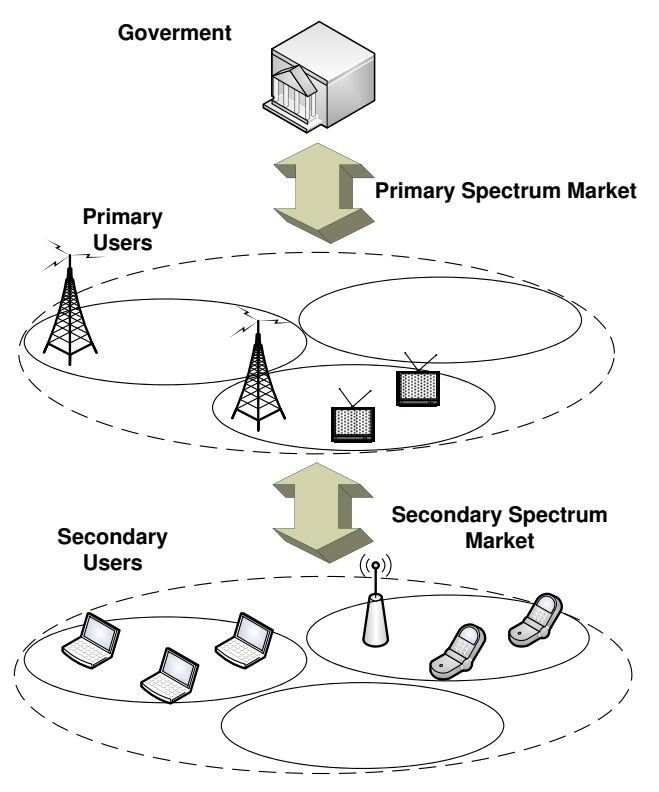

Figure 1: Spectrum market structure.

is sufficient incentive, current licensed spectrum holders are likely to be reluctant to allow open access, and trying to achieve this purely through policymaking and regulation could be problematic. At the same time, we envision the emergence of various secondary users whose spectrum needs may be highly variable, sporadic and short lived, and limited to specific geographical regions.

The above observations have motivated us to consider a market mechanism particularly targeted at spectrum access relationship between primary users and secondary users (service providers), as shown in Figure 1. In this paper, we present a framework in which these secondary users could tap into unused or under-used spectrum bands currently held by primary users, while the latter can generate additional profit in the process. One of the key features of this market is that the spectrum contracts cover relatively small geographic regions, such that purchase of one contract may preclude the sales of the same spectrum bands in neighboring regions, while spectrum can be reused in non-interfering regions.

We will use auction as the form of trading in this system and will refer to this as a secondary spectrum market for spectrum access. There are a number of desired properties associated with such an auction system, including truthfulness, high revenue for the sellers and high spectrum resource utilization for the system as a whole. As we will demonstrate, our framework addresses all these aspects. It has to be mentioned that auction methods have been quite extensively used in the context of spectrum access and sharing, see e.g., [7]. More on related work is discussed in Section 8. Our work is different from in that we focus on revenue generation of truthful auctions whiling considering the interference nature of wireless communication.

We begin by presenting a number of potential bidding formats in this framework. We then focus on one particular implementation of the system corresponding to the following type of bidding format: Each buyer is interested in purchasing access to a certain number of channels in each of a fixed set of cells (i.e., geographical locations); their requests are strict (i.e., cannot be partially satisfied). We design auction mechanisms that maximize expected revenue while ensuring truthful bidding from buyers. Assuming that the buyers' valuation distributions are known to the seller, we first design an optimal auction based on using the Vickrey-ClarkeGroves (VCG) mechanism to enforce truthfulness and maximize expected revenue. Here we adopt the concept of virtual valuation instead of the buyers' original value, which is the surplus of the original valuation and a function of valuation distribution. This method is widely used in conventional economical analysis. It can be shown that maximizing total virtual valuation is equivalent to maximizing expected revenue. However, the winner determining problem with the VCG mechanism in the graph structured resource is a NPHard problem. Therefore, we also provide a suboptimal auction in terms of revenue generation, but that addresses the complexity of maximization computation. This is achieved by using a greedy-like allocation and a critical value based payment rule, which together induce truthful bidding. Its low complexity makes this auction much more practical for spectrum auctions on a shorter time scale. We examine the effectiveness of this method via numerical results.

The main contribution of this paper is as follows:

1. We investigate the revenue generation problem for primary users in a secondary spectrum auction market, where truthful bidding and interference constraints are satisfied.

2. Under the Baysian setting, we use the VCG auction to maximize the expected revenue by maximizing the associated virtual valuation, under the interference constraints. We also propose a suboptimal auction to reduce the complexity for practical purpose.

The rest of the paper is structured as follows. In Section 2 we characterize the special challenges in a secondary spectrum market. Section 3 formally presents the spectrum auction market. We then consider a specific case in Section 4. Sections 5 and 6 present two types of auctions, respectively. Experimental results are shown in Section 7. Section 8 reviews related work. We conclude the paper in Section 9.

\section{CHARACTERIZATION OF SECONDARY SPECTRUM MARKET}

In this section we describe at a conceptual level of secondary spectrum market and characterize its uniqueness which makes it different from traditional (primary) spectrum market and motivates our research. As pointed out in the introduction, our objective is to provide a market and trading mechanism for both primary license holders (seller) and secondary wireless service providers (buyers). Specifically, through such a mechanism the former can profit from selling shorter-term (e.g., weeks or months) spectrum licenses/ contracts, while their own licenses, issued in the traditional spectrum acquisition procedure, are typically valid for a much longer period. Such short-term licenses are on a much finer granularity in terms of the amount of spectrum and the area coverage each such license applies to, which is different from the traditional, coarse-grained spectrum assignment. This mechanism provides a clear incentive for the primary spectrum holders to allow dynamic access to under-used 
spectrum opportunity. It will also undoubtedly improve the overall spectrum utilization and efficiency.

\subsection{Revenue Generation}

We first note that in a traditional primary spectrum market, the government's key objective is to efficiently assign the spectrum resource to users with the greatest valuation, so that social welfare may be maximized. By contrast, in a secondary spectrum market, incentive becomes a critical issue. Many primary users are private companies driven by self-interest. Their spectrum bands are obtained via traditional spectrum auctions, at (sometimes significant) costs. Their natural goal is to maximize their own profit. Without sufficient incentive, primary users are likely reluctant to open their spectrum bands to other users.

It follows that primary users, in opening access to their spectrum bands, will try to optimize their revenue by using appropriate allocation and pricing rules. This is the main issue we try to address in this paper. In subsequent sections we will primarily focus on using auction as the underlying trading mechanism, where there is only one spectrum holder and potentially many spectrum buyers.

\subsection{Finer Spectrum Reuse}

There are two key assumptions underlying the proposed spectrum market. The first one is that this primary license holder has fairly accurate information on the spectrum usage within its band and its coverage. Such information may be obtained from statistics collected during operation or extensive spectral measurement. From such information it may deduce that certain sections of its spectrum in certain regions within its coverage during certain periods of time (or time of day or days of the week, etc.) are grossly underutilized, and therefore would like to lease them out for profit. A second assumption is that the effect time of contracts in the market are at least on the order of hours, rather than seconds or less. Two cases can be identified further: (1) the primary users do not show up during the whole period of the contracts, so secondary wireless service providers can use the spectrum freely [8]. (2) There are some primary activities. Secondary wireless service providers must obey certain rules to avoid harmful interference, which many studies on opportunistic spectrum access on the packet level seek to exploit [9]. However, in designing auctioning mechanisms we need not be concerned with the distinction between these two cases.

These spectrum opportunities can be location-dependent; highly populated urban areas may only have limited spectrum to spare while vast white spaces exist over some rural areas. They can also be time-dependent. For a certain area, more spectrum may be available in one period of time than the other. With a collection of such spectrum opportunities, the primary license holder can now formally define a number of spectrum-space-time blocks/units, and generate a contract for each of them.

\section{SECONDARY SPECTRUM AUCTION FRAMEWORK}

In this section we describe in detail our spectrum auction framework. The design of the framework has to take into account a number of objectives, including spectrum usage efficiency, flexible spectrum requests and achievable auction properties including truth-revelation and revenue maximization. While some of these objectives are certainly correlated, in general they cannot all be achieved at the same time. Our framework consists of the following elements: spectrum resource, bidding language and auction objective.

\subsection{Spectrum Resource}

Radio spectrum is a special resource with multi-dimensional properties (space, time and frequency) [10] [11]. Within the context of this paper, we will ignore the time dimension by assuming that time is partitioned into periods and that the auction under consideration is limited to one such period. Implicitly, spectrum demand (bids submitted) and availability (supply of contracts) remain constant within a period. With this simplification, the primary license holder organizes its spectrum availability into certain frequency-space units.

In general it is up to the primary license holder to decide on a sensible and potentially profitable way to organize its spectrum availability into concrete, sellable frequency-space units. For instance, it may choose to first divide its spectrum into groups based on different spectral characteristics, and hold auctions separately for each group. At the same time, the primary license holder also partitions its coverage area, where there are available frequency bands, into cells. This cell partition process may heavily depend on regional geographic and demographic properties. It is very likely that cells will have different sizes. This process also depends on the spectral characteristics. For example, if we consider the TV band channels, the size of each cell can have a radius of 10 to $100 \mathrm{~km}$.

For the purpose of our analysis, we will focus on a single spectrum group that is divided into a number of bands of equal bandwidth. We assume that for this set of frequency bands the corresponding cells are given. In some of those cells spectrum may be reused while in others (neighboring cells) we must use different bands. Each secondary spectrum contract specifies a pair of frequency band (i.e., channel) and cell.

These assumptions allow us to represent the spatial reuse relationship of cells using an interference graph, where each node corresponds to a single cell, and an edge between two nodes indicates that the connecting cells interfere with each other when the same band is used in both. The auction market essentially tries to achieve the following: through a bidding and pricing process the system determines on this graph which frequency bands are used in which cell by which buyer. Regardless of other elements of the auction market, the resulting frequency allocation has to be interference free, i.e., no two neighboring nodes can be allocated the same channel.

\subsection{Bidding Language}

Buyers can express different formats of spectrum request depending on the type of application scenarios involved and their own interest. Below we list several representative spectrum request formats. We use $B_{i}$ to denote the submitted bid of buyer $i$, which takes on different format in each case.

\subsubsection{Total Channels in All Cells}

Each buyer submits its bid $B_{i}$, indicating its total requested number of channels across all cells, and its bidding price. In this case, buyers care more about the total amount 
of spectrum it can get; it does not care where and at what density the allocated spectrum lies. In some situations, the requirement can be partially accepted, while in other cases, the requirements are strict.

An example where this bidding format makes sense is a wireless service provider with fairly widely deployed infrastructure. It has a large overall spectrum demand and wants to acquire as much spectrum as possible regardless of the locations and spectrum distributions.

\subsubsection{Cells and Channels per Cell}

Each buyer submits its bid $B_{i}$ indicating the requested number of cells, the requested number of channels in each cell, and the total bidding price. Both strict and partial requirements are possible.

A motivating example for this format is a wireless service provider with well deployed infrastructure over a whole region, and its spectrum demand for each sub-region tends to be uniform. Due to practical issues such as a budget constraint, it would like to have some cells upgraded but does not care which cells these are. Another case is when a wireless service provider wants to deploy new services in a region with service coverage requirement (e.g., coverage portion of the whole region) and spectrum density requirement in each cell.

\subsubsection{Specific Cells and Corresponding Channels}

Each buyer requests a certain number of channels in fixed cells and a total bidding price. Both strict and partial requirements are possible.

This case is suitable when the buyers have particular interest in specific locations. This possibility of preference can be easily understood when a provider considers where to initiate certain services with a limited budget (e.g., downtown vs. suburbs). This also applies to scenarios where a service provider is bound to specific areas and locations due to practical or regulatory constraints.

\subsection{Desired Properties of Spectrum Auction}

From the seller's point of view, the spectrum auction ideally should fulfill certain objectives, such as revenue generation, truthful biding and computational efficiency.

\subsubsection{Revenue}

As already mentioned, the spectrum seller naturally would like to maximize its potential revenue given the bids from buyers. If the seller does not care about whether the bids are truthful, then this leads to a revenue maximization problem. Obviously, the price charged for winning buyers must be no greater than their bidding price. An efficient resource allocation algorithm is then needed for the revenue maximization.

\subsubsection{Truthfulness}

The seller may also desire truthful bids from buyers. Truthtelling prevents market manipulation, and eases the bidding of buyers since bidding with their true value is the best strategy in theory. Single-unit second price auction and multipleunit Vickery-Clarke-Groves (VCG) mechanism are the typical rules to enforce truthfulness.

\subsubsection{Computational Efficiency}

In some situations when the amount of resources is large or the time granularity is small, the auction needs to be efficient in that the computational complexity needs to be low to work in real-time or near real-time. For example, even though the VCG mechanism can enforce truthfulness, its computational complexity is high in general.

\section{AUCTION MODEL FOR REQUEST OF SPECIFIC CELLS AND CORRESPOND- ING CHANNELS}

We now focus on a particular spectrum auction bidding format mentioned in the previous part, the Specific Cells and Corresponding Channels format. The buyers have spectrum demand for particular cells and a certain number of channels for each cell (demand for each cell may be different). The auction is a single sealed bid auction. Strict request is adopted here, which means the winners get either all requested spectrum or nothing.

We first describe our assumptions and notations for the spectrum auction. The seller offers $K$ channels covering a certain region. The channels have uniform characteristics, therefore buyers will not differentiate between channels. The region is partitioned into small cells. If adjacent cells use the same channel, they will interfere with each other. The space is represented by a graph $G=(\mathbf{V}, \mathbf{E})$, where $\mathbf{V}$ denotes the set of cells with $M=|\mathbf{V}|$, and $\mathbf{E}=\left\{e_{j j^{\prime}}\right\}_{M \times M}$ denotes the interference relation among cells, with $e_{j j^{\prime}}=1$ indicating there is interference between cell $j$ and $j^{\prime}$, and 0 otherwise. The graph $G$ is assumed to be the same for all the channels.

There are $N$ buyers participating in the spectrum auction. Each of them submits a bid $B_{i}=\left(\mathbf{d}_{i}, b_{i}\right)$, where $\mathbf{d}_{i}$ is the spectrum demand and $b_{i}$ is the bidding price for the submitted demand. Here $\mathbf{d}_{i}=\left(d_{i}^{1}, \ldots, d_{i}^{M}\right)$ is a demand vector with $d_{i}^{j}$ representing the number of channels buyer $i$ demands in cell $j$.

We follow the conventional Bayesian setting in economics. We use $v_{i}$ to denote the privately known valuation of buyer $i$ for spectrum resource $\mathbf{d}_{i}$. We assume the exact valuation $v_{i}$ is private information, but the distribution $F_{i}$ from which $v_{i}$ is drawn is known to the seller; while buyers have no knowledge of each other's valuation distribution. The corresponding density function is $f_{i}=\frac{d}{d z} F_{i}(z)$, which is continuous. Such information can be based on the records of history transactions.

Given the bids from buyers, the seller computes the outcome consisting of a winner determining vector $\mathbf{x}=\left(x_{1}, \ldots, x_{N}\right)$ and charging prices $\mathbf{p}=\left(p_{1}, \ldots, p_{N}\right)$, where $x_{i}=1$ means buyer $i$ wins and is allocated with its whole spectrum request while $x_{i}=0$ is for no allocation for buyer $i$ at all, $p_{i}$ is the amount of money buyer $i$ is intended to pay the seller. The utility obtained by buyer $i$ is indicated by $u_{i}=v_{i} x_{i}-p_{i}$.

As we have mentioned earlier, even though in reality a winning bidder may or may not have exclusive use to the channels he gains access to, i.e., he may or may not have to share with the primary user (seller), the above auction model does not distinguish between the two and can be applied equally in either case. This is because this model does not concern the finer details of the terms of usage specified in the contracts, and by submitting bid $B_{i}$ a buyer agrees to abide by such terms, whatever they are.

The winner determination vector $\mathbf{x}$ should be associated with a feasible allocation, which is denoted by a vector $\mathbf{A}=\left(\mathbf{A}_{1}, \ldots, \mathbf{A}_{N}\right)$, where $\mathbf{A}_{i}=\left\{a_{i}^{j k}\right\}_{M \times K}$ is an allocation matrix for buyer $i$ with $a_{i}^{j k}=1$ meaning channel $k$ 
at cell $j$ is allocated to buyer $i$ and $a_{i}^{j k}=0$ otherwise. It is up to the seller to allocate the spectrum without mutual interference, i.e., the same channel is not allocated to cells with connecting edges. Formally, the constraints for a feasible winner determination and its allocation vector $\mathbf{A}$ can be expressed as follows:

Allocation Constraints:

$$
\begin{array}{r}
\sum_{k=1}^{K} a_{i}^{j k}=x_{i} d_{i}^{j}, \quad j=1, \ldots, M, i=1, \ldots, N \\
\sum_{i=1}^{N} a_{i}^{j k} \leq 1, \quad j=1, \ldots, M, k=1, \ldots, K \\
\sum_{i=1}^{N} a_{i}^{j k} \cdot \sum_{i=1}^{N} a_{i}^{j^{\prime} k}=0, \quad \forall e_{j j^{\prime}}=1, k=1, \ldots, K
\end{array}
$$

where (1) ensures the allocation for buyers is strict, (2) indicates that a given channel in a given cell can only be allocated to at most one buyer, and (3) is the interference constraint.

DEFINITION 1. An auction is truthful if and only if buyer $i$ 's (expected) utility of bidding its true valuation $v_{i}$ is at least its (expected) utility of bidding any other value $b_{i}$,

$$
u_{i}\left(v_{i}, \mathbf{b}_{-i}\right) \geq u_{i}\left(b_{i}, \mathbf{b}_{-i}\right) .
$$

The expected revenue of the primary user is given by:

$$
E[\pi]=E\left[\sum_{i=1}^{N} p_{i} x_{i}\right],
$$

where the valuation $v_{i}$ follows a known distribution $F_{i}\left(v_{i}\right)$, the winner determination $\mathrm{x}$ subject to the Allocation Constraints, and the auction is truthful.

We use $\mathbf{b}_{-i}=\left(b_{1}, \ldots, b_{i-1}, b_{i+1}, \ldots, b_{N}\right)$ to represent the vector of bidding prices without buyer $i$ 's bid, and also similar for $\mathbf{x}_{-i}$.

In the next two sections, we will present two auction designs: an optimal auction with high computational complexity, and a computational efficient auction with suboptimal revenue.

\section{OPTIMAL AUCTION}

In this section, we present an optimal auction which maximizes the expected revenue. We use the conventional economics approach of Bayesian optimal mechanism design, where it is assumed that the valuations of buyers are drawn from distributions known to the seller. We base our auction on Myerson's optimal mechanism, which is a truthful mechanism that maximizes the auctioneer's expected revenue. With the knowledge of valuation distribution, the notion of virtual valuation is defined, which is essentially a reservation price for each buyer. We apply VCG mechanism on the virtual valuation, which generates the maximum expected revenue while enforcing truthfulness. However, the computational complexity is shown to be high.

\subsection{Virtual Valuation}

We first present the concept of virtual valuation from [12].

DeFinition 2. The virtual valuation of buyer $i$ with valuation $v_{i}$ is

$$
\phi_{i}\left(v_{i}\right)=v_{i}-\frac{1-F_{i}\left(v_{i}\right)}{f_{i}\left(v_{i}\right)} .
$$

We assume the distribution of valuation $v_{i}$ satisfy the monotone hazard rate assumption: $\frac{f_{i}(z)}{1-F(z)}$ is monotone nondecreasing. With such an assumption, the virtual valuation function is a monotone non-decreasing function. In doing so the mechanism discriminates the buyers: associating high reservation price with bidders with high valuation distribution while low reservation price with bidders with low valuation distribution.

\subsection{VCG-based Auction}

With the above virtual valuations, the revenue maximization problem can be solved with the well known VCG mechanism: the winner determination is to maximize the sum of winning bids and the payment from each buyer is the opportunity cost that its presence introduces to all the other players. The detailed auction is as follows:

1. Given the bids $\left\{B_{i}\right\}$ and $\left\{F_{i}\right\}$, compute virtual bids $B_{i}^{\prime}=\left(\mathbf{d}_{i}^{\prime}, b_{i}^{\prime}\right)$ where $\mathbf{d}_{i}^{\prime}=\mathbf{d}_{i}$ and $b_{i}^{\prime}=\phi_{i}\left(b_{i}\right)$.

2. Use VCG mechanism on the virtual bids to get $\mathbf{x}^{\prime}$ and $\mathbf{p}^{\prime}$.

VCG mechanism includes an allocation to maximize the virtual valuation of all the users:

$$
\begin{aligned}
& \max _{\mathbf{x}^{\prime}} \sum_{i=1}^{N} \phi_{i}\left(b_{i}\right) x_{i}^{\prime} \\
& \text { s.t. Allocation Constraints. }
\end{aligned}
$$

VCG mechanism results in the payment:

$$
p_{i}^{\prime}=\max _{\mathbf{x}_{-i}} \sum_{j \neq i} x_{j} \phi_{j}\left(b_{j}\right)-\max _{\mathbf{x}} \sum_{j \neq i} x_{j} \phi_{j}\left(b_{j}\right) .
$$

3. The final allocation $\mathbf{x}$ is set to $\mathbf{x}^{\prime}$ and the charged price $\mathbf{p}$ is set with $p_{i}=\phi_{i}^{-1}\left(p_{i}^{\prime}\right)$ for each winner and $p_{i}=0$ for each loser.

\subsection{Properties of the Optimal Auction}

We now analyze the properties of the optimal auction in terms of truthfulness, revenue and complexity. It is known that an auction is truthful if the allocation algorithm of this auction is monotone while the price charged of a winner is a critical value [13]:

1. Monotone allocation: for each $\mathbf{b}_{-i}$, if buyer $i$ wins by bidding $b_{i}$, then it also wins by bidding $b_{i}^{\prime}+\delta$ with $\delta>0$.

2. Critical value: monotone allocation implies there is a critical value $v_{i}^{c}$ for each buyer such that if buyer $i$ bids higher than $v_{i}^{c}$ it wins and if $i$ bids lower than $v_{i}^{c}$ it loses.

We show that the allocation of the optimal auction is indeed a monotone allocation despite the interference constraints from spectrum resource. The requirement of satisfying the interference constraints is not present in existing literatures of auction design [13].

LEMMA 1. The winner determination $\mathbf{x}$ induced by virtual valuation maximization is monotone non-decreasing, if distribution function $F_{i}(z)$ satisfies the condition that $\frac{1-F_{i}(z)}{f_{i}(z)}$ is non-increasing. 
Proof. Suppose $\mathbf{x}$ is a winner determination vector which maximizes the sum of virtual valuations $S=\sum_{\mathbf{x}} \phi_{i}\left(b_{i}\right) x_{i}$ subject to the Allocation Constraints, with buyer $i$ is allocated with its request $\mathbf{d}_{i}$, i.e., $x_{i}=1$. Suppose buyer $i$ 's bidding price is increased from $b_{i}$ to $b_{i}+\delta$ with $\delta>0$ while the other buyers keep their bidding prices $\mathbf{b}_{-i}$ unchanged. With the assumption of monotone hazard rate, we have $\phi_{i}\left(b_{i}+\delta\right)>\phi_{i}\left(b_{i}\right)$. Then to maximize $S$, it must be the case that $x_{i}^{\prime}=1$.

According to the pricing rule of the optimal auction, i.e., Equation (8), the (virtual) payment for a winner does not depend on its bidding price, which means it is a critical value. Therefore the requirement of critical value is satisfied immediately.

LEMMA 2. If buyer $i$ wins, its payment $p_{i}$ is a critical value.

From Lemma 1 and 2, we can get the following result:

THEOREM 1. The optimal auction is truthful in that each buyer maximizes its utility by submitting its truthful valuation.

Proof. Let $v_{i}$ be the truthful bidding, and $b_{i}$ the bidding such that $b_{i} \neq v_{i}$.

In the first case, if buyer $i$ loses by bidding $b_{i}$, then its utility $u_{i}\left(b_{i}\right)=0$. While bidding $v_{i}$ brings it nonnegative utility $u_{i}\left(v_{i}\right) \geq 0$, with $u_{i}\left(v_{i}\right)=0$ for losing and $u_{i}\left(v_{i}\right)=$ $v_{i}-p_{i} \geq 0$ for winning.

In the second case, buyer $i$ wins by bidding $b_{i}$. If buyer $i$ also wins with $v_{i}$, the utilities are the same, since the payment of critical value is the same, i.e., $u_{i}\left(v_{i}\right)=u_{i}\left(b_{i}\right)=$ $v_{i}-p_{i}$. If buyer $i$ loses with $v_{i}$, according to the monotonicity property, it must be the case of $v_{i} \leq p_{i} \leq b_{i}$. Then the utility for bidding $b_{i}$ is negative, i.e., $u_{i}\left(b_{i}\right)=v_{i}-p_{i}<0$.

In both cases, bidding other than truth valuation is not better than bidding truth valuation.

The proof is similar to the one in [13].

For the expected revenue, it is shown in [12] that for any truthful mechanism and fixed bids $\mathbf{b}_{-i}$ of all bidders except for bidder $i$, the expected payment from a bidder $i$ satisfies:

$$
E\left[p_{i}\left(b_{i}\right)\right]=E\left[\phi_{i}\left(b_{i}\right) x_{i}\left(b_{i}\right)\right] .
$$

Therefore, to maximize the expected revenue of a truthful auction, it suffices to maximize the total virtual valuations.

THEOREM 2. The optimal auction maximizes the expected revenue.

Since the expected revenue of any truthful mechanism is equal to the expected total virtual valuation, and the optimal auction is indeed maximizing the total virtual valuation, the optimal auction maximizes the expected revenue.

\section{THEOREM 3. The VCG-based optimal auction is NP-hard}

Proof. We check the winner determining problem in the optimal auction. For the simple case that there is one single channel and each buyer's submission contains one single cell, the virtual valuation maximization is a weighted independent set problem, which is an NP-Hard problem.

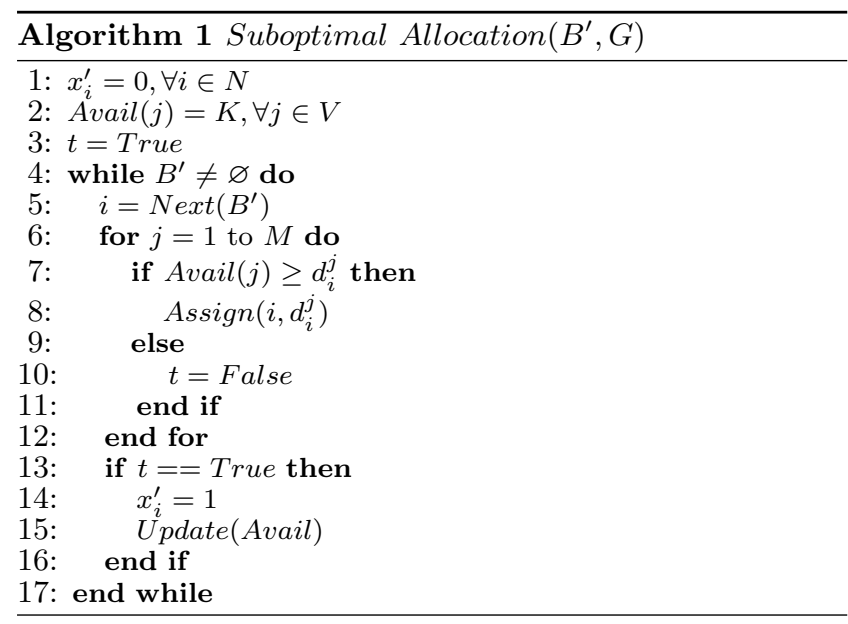

\section{SUBOPTIMAL AUCTION}

The VCG-based optimal auction introduced in the previous section is able to generate maximum expected revenue, but the computational complexity is prohibitive for large scale spectrum market and real-time spectrum trading. Therefore, in this section, we further propose a more computationally efficient auction. The low complexity comes at the price of some reduction in revenue, but as we will show this suboptimal auction preserves the truthful property.

\subsection{Non-VCG Auction}

One intuitive approach to address the issue of complexity is to use an approximate algorithm for the winner determination problem and a VCG-like pricing algorithm. However, it has been reported that such an approach cannot enforce truthful bidding in general. We therefore resort to one type of non-VCG auction [14], which also consists of three steps: bid ranking, winner determination along with spectrum allocation, and payment calculation. This will be referred to as the suboptimal auction. The complete auction rule is described as follows:

1. Compute virtual bids $B_{i}^{\prime}=\left(\mathbf{d}_{i}^{\prime}, b_{i}^{\prime}\right)$ as in the optimal auction: $\mathbf{d}_{i}^{\prime}=\mathbf{d}_{i}$ and $b_{i}^{\prime}=\phi_{i}\left(b_{i}\right)$.

2. Use the non-VCG mechanism on the virtual bids to get $\mathbf{x}^{\prime}$ and $\mathbf{p}^{\prime}$, which includes:

a) In the first step of bid ranking, the bids are sorted by some criterion: $r(i)=r\left(\mathbf{d}_{i}^{\prime}, b_{i}^{\prime}\right)$. The sorted list with deceasing order is denoted by $L$. Different rankings can be used here as long as it satisfies monotonicity. In this paper, we use the metric of average virtual valuation per requested cell: $r(i)=\frac{b_{i}^{\prime}}{s_{i}\left(\mathbf{d}_{i}^{\prime}\right)}$, where $s_{i}\left(\mathbf{d}_{i}^{\prime}\right)$ is the total number of demanded channels in all cells: $s_{i}\left(\mathbf{d}_{i}^{\prime}\right)=\sum_{j=1}^{M} d_{i}^{j}$.

b) In the second step, a greedy algorithm generates an allocation with the skeleton shown in Algorithm 6.1. In each iteration, the algorithm checks the next bid on the list $L$. It iteratively checks the demand for each cell in the order of cell index: during the iteration, it allocates the demanded number of channels in the cell in the order of channel index if there are enough channels in the cell, and updates the channel availability in 


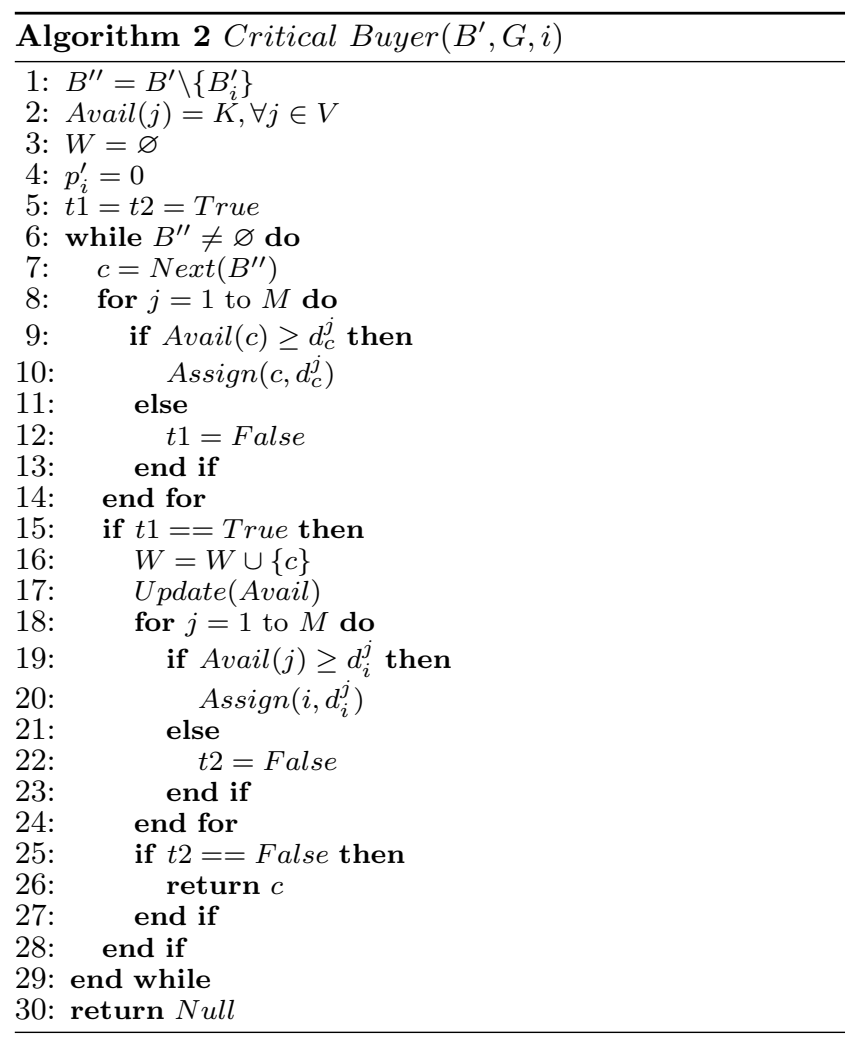

neighboring cells. The algorithm grants the whole request of bid $i$ if it does not conflict with any allocation previously granted (i.e., the Allocation Constraint). If there is confliction, it denies (i.e., does not grant) the bid.

c) In the third, payment step, a buyer $i$ pays the amount according to a critical buyer $c(i)$ such that: if buyer $i$ 's bid satisfies $r(i)>r(c(i))$, buyer $i$ wins; if $r(i)<r(c(i))$, it loses. The critical buyer $c(i)$ for buyer $i$ can be determined by Algorithm 2. Intuitively, the algorithm finds the first buyer who makes buyer $i$ 's demand unsatisfied. The virtual payment is the average virtual valuation per cell of buyer $c(i)$ multiplied by the requested amount of buyer $i: p_{i}^{\prime}=r(c(i)) \cdot s_{i}\left(\mathbf{d}_{i}^{\prime}\right)$.

3. The final allocation $\mathbf{x}$ is set to $\mathbf{x}^{\prime}$ and the charged price $\mathbf{p}$ is set to $p_{i}=\phi_{i}^{-1}\left(p_{i}^{\prime}\right)$ for each winner and $p_{i}=0$ for each loser.

Note that the allocation algorithm is different from typical greedy algorithms in that the allocation order in the former is fixed in the beginning and will not change during the whole allocation process.

\subsection{Properties}

We examine properties of the above auction in terms of truthfulness and complexity. The result of revenue is given in the next section.

LEMMA 3. The allocation resulting from the suboptimal auction is monotonic.

Proof. Suppose we increase buyer $i$ 's bid from $b_{i}$ to $b_{i}+\delta$ with $\delta>0$. By our definition of bid ranking, we have $r\left(b_{i}+\right.$

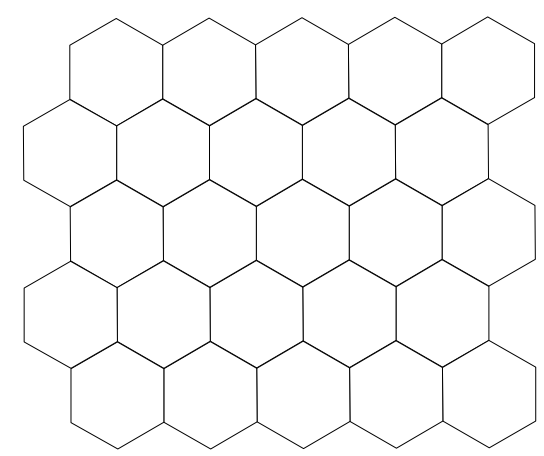

Figure 2: Cellular topology with 25 cells.

$\delta) \geq r\left(b_{i}\right)$. Denote the lists for $b_{i}$ and $b_{i}+\delta$ as $L$ and $L^{\prime}$, respectively. If we compare the lists $L$ and $L^{\prime}$ obtained, we see that they differ only in that $i$ 's bid may have been moved up the list by changing from $b_{i}$ to $b_{i}+\delta$. The allocation algorithm performs (i.e., grants or denies bids) in exactly the same way on $L$ and $L^{\prime}$ until it reaches $i$ 's bid in $L^{\prime}$. Assume $i$ 's bid is denied on $L^{\prime}$, which implies that there are not enough channels among $i$ 's requested cells, either because $i$ 's request is not feasible, or because some other bids on $L^{\prime}$ before $i$ 's are granted with conflicting cells (collocated or interfering). The same reasoning also applies to $i$ 's bid on $L$ and thus it would also be denied. Now assume $i$ 's bid on $L$ is granted, which means that $i$ 's bid is feasible and bids granted before $i$ (if any) leave enough channels in $i$ 's requested cells. Again this is also true for $i$ 's bid on $L^{\prime}$ and thus $i$ 's bid is also granted on $L^{\prime}$.

LEMMA 4. Each winner in the suboptimal auction pays the its critical value $v_{i}^{c}$ according to $c(i)$.

Proof. This is obvious since the virtual payment $p_{i}^{\prime}$ is based on the critical buyer $c(i)$ according to Algorithm 2 . With the mapping between value and virtual value, the final payment is also a critical value.

With Lemmas 3 and 4, we obtain:

TheOREM 4. The suboptimal auction is truthful.

The proof is similar to the truthfulness proof in the previous section.

THEOREM 5. The suboptimal auction has a polynomialtime complexity. It runs in time $O(N \log N+M N K|\mathbf{E}|)$, where $|\mathbf{E}|$ is the number of edges in the graph $G, M$ is the number of cells, $N$ is the number of bidders, and $K$ is the number of channels auctioned.

\section{EXPERIMENTAL RESULTS}

In this section we use experiment results to evaluate the performance of the proposed auctions. We use a cellular system-like topology shown in Figure 2. There are 25 cells with equal size. The number of buyers is fixed at 5 . The interference range of a cell is limited to its immediate neighborhood. Each buyer requests at most one channel in any cell in the topology, and each buyer's valuation for its demand follows either the uniform distribution $F(z)=z$ in the range of $(0,1]$, or the exponential distribution $F(z)=1-e^{-\lambda z}$ with 


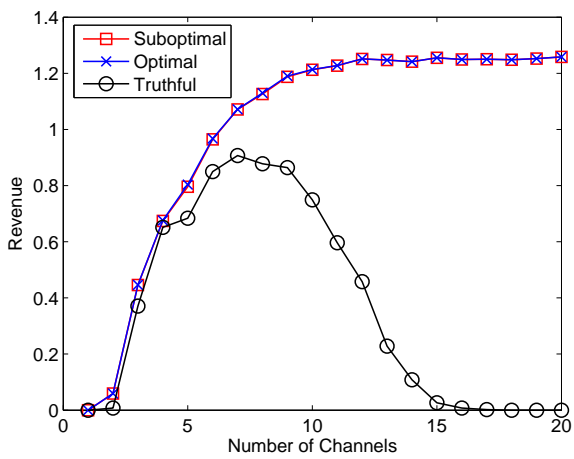

(a) Revenue

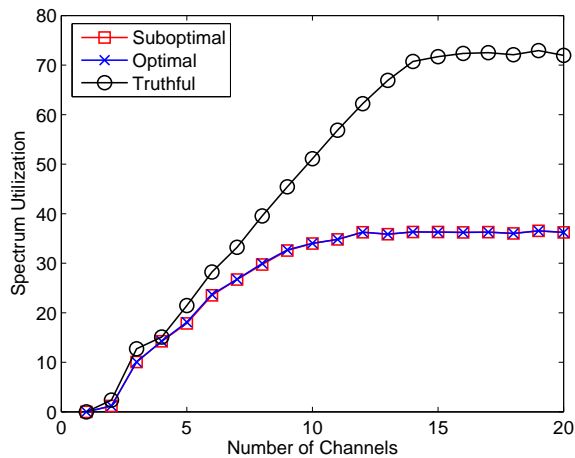

(b) Spectrum utilization

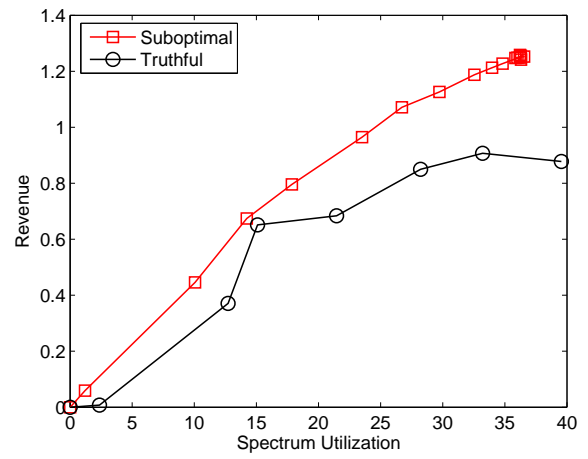

(c) Revenue vs. Spectrum utilization

Figure 3: (Uniform distribution) Comparison between the suboptimal auction, optimal auction and truthful auction in terms of: (a) revenue (b) spectrum utilization (c) revenue vs. spectrum utilization

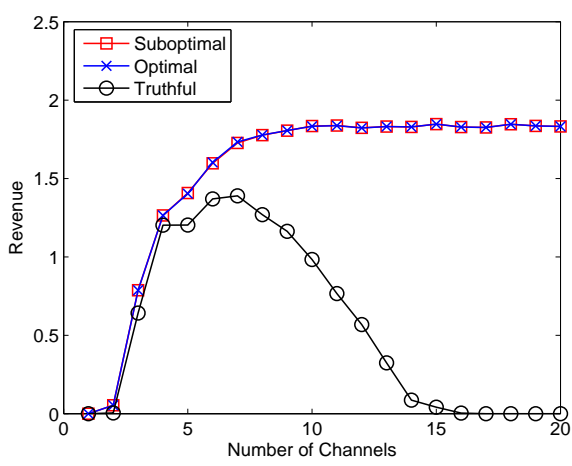

(a) Revenue

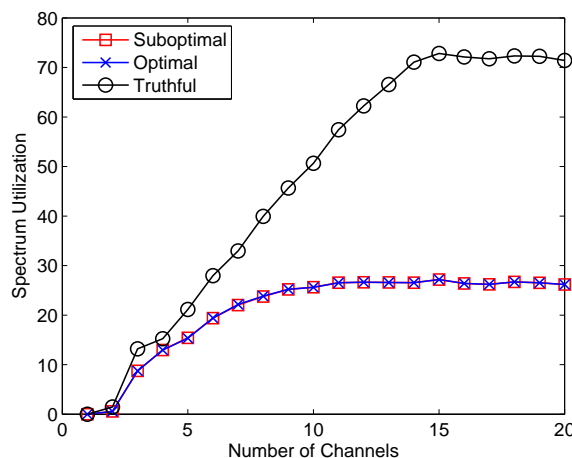

(b) Spectrum utilization

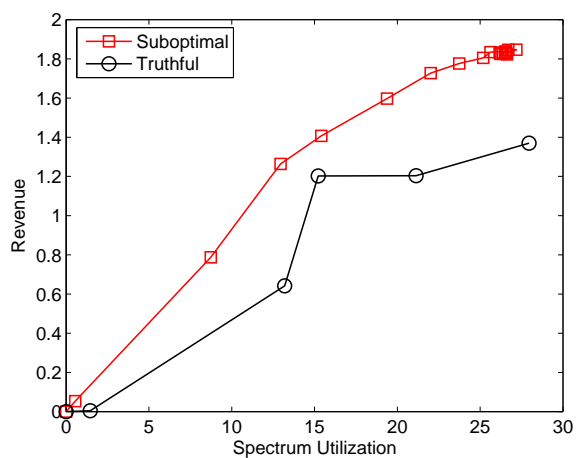

(c) Revenue vs. Spectrum utilization

Figure 4: (Exponential distribution) Comparison between the suboptimal auction, optimal auction and truthful auction in terms of (a) revenue (b) spectrum utilization (c) revenue vs. spectrum utilization

$\lambda=1$. For each random realization of demands, results are averaged over 100 runs to obtain the expected results for different biddings. The final results are averaged over 100 randomly generated demands. The results are presented as the number of channels available for auction increases.

We compare the performance of the suboptimal auction, optimal auction and another auction (labeled as truthful in the figures). The third auction is a truthful auction without considering the valuation distribution: it is similar to the suboptimal auction but it uses valuation instead of virtual valuation. We refer to this auction as the truthful auction. A special case of this auction where each buyer can only request a single location is discussed in [15].

We first consider the uniform distribution. As shown in Figure 3(a), the suboptimal auction and optimal auction outperform the truthful auction in terms of revenue. The truthful auction's revenue suffers significant degradation as spectrum supply increases and eventually decreases to zero. This is because when supply increases, the number of losers decreases, and in turn payments from winners also decreases. In contrast, the suboptimal and optimal auction generate stable revenue as the spectrum supply increases. This is due to the reservation effect of virtual valuation. This reservation is clearly shown in Figures 3(b) and 3(c): as shown, the suboptimal can generate more revenue than the truthful auction with equal amount of allocated spectrum resources. Further more, the performance of suboptimal auction is approaching the optimal auction. Considering the lower complexity, the suboptimal auction is more preferable.

Figure 4 displays the results from using the exponential distribution, which are similar to the uniform one. We conclude from these results that the suboptimal auction is a good candidate in terms of revenue generation.

We present the social welfare, which is defined as the total valuation of the winners: $\sum_{i=1}^{N} v_{i} x_{i}$. We only compare the social welfare of suboptimal auction with the one of VCG auction (with uniform distribution of valuation), considering the similar results of suboptimal and optimal auction. VCG auction is known to maximize the social welfare. As shown in Figure 5, there exist a gap between the social welfare of suboptimal and VCG auction, since the spectrum resource is not fully allocated to buyers with suboptimal auction. Therefore, the spectrum holder's revenue maximization is at the cost of degradation of social welfare.

We also tested the auctions in terms of certain properties of spectrum requests. Specifically, we consider two types of requests: scattered requests and clustered requests. In both cases, the number of total demanded channels in each cell is the same. For scattered requests, the request of each buyer is scattered over the entire region. For clustered re- 


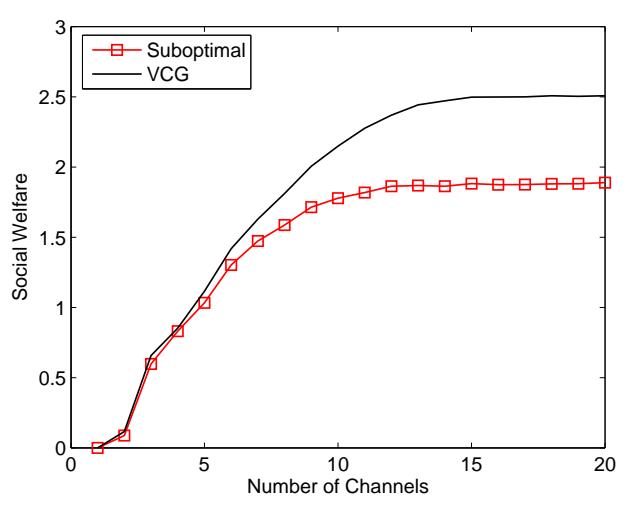

Figure 5: Social welfare for suboptimal auction and VCG auction.

quests, the request of each buyer is clustered within a small region while the location of such a small region is random. As shown in Figure 6, the suboptimal auction outperforms the truthful auction in terms of revenue for both requests. Furthermore, with clustered requests, the revenue decreases because mutual interference limits the allocation and less of buyer's requests are satisfied.

\section{RELATED WORK}

General auction theory has been studied for decades. Efficient auctions, e.g, VCG based auction, are known to maximize social welfare. Optimal auctions [12] target maximizing revenue, a concept used in this paper. However, the auctioned resource here is not a single object, but a group of cell and channel resource with mutual interference relationship. Complexity issues of VCG auction are studied in $[14,16]$ which target social welfare. We extend them into the graph structured resource and use them to address the expected revenue issue.

It has long been recognized that compared to approaches such as comparative hearings and lotteries, market-based mechanisms are more promising and potentially far more efficient for spectrum allocation. There are comprehensive literatures related to the traditional spectrum auction $[17,18]$. We emphasize however that these spectrum auctions aim at allocations on a much bigger scale than that considered in this paper. More particularly, the interference and spatial reuse characteristic of spectrum are not considered in those works.

Several studies within the context of spectrum sharing consider the unique characteristic of spectrum resources. [15] proposes a general spectrum auction framework considering the interference relationship in spectrum spatial reuse. A strategy proof spectrum auction market is proposed and high spectrum utilization is achieved. The intended buyers in this market are end users (transmitters) or clustered groups of users, each is only interested in spectrum availability in its own fixed location. [19] proposes a multi-winner auction to optimize spectrum efficiency and prevent collusion, while the truthful bidding is compromised. [20] proposes approximation algorithms to approach the optimal revenue for spectrum holder subject to interference constraints. However, they do not consider the truthfulness of buyers. [21] uses auction to allocate transmit power

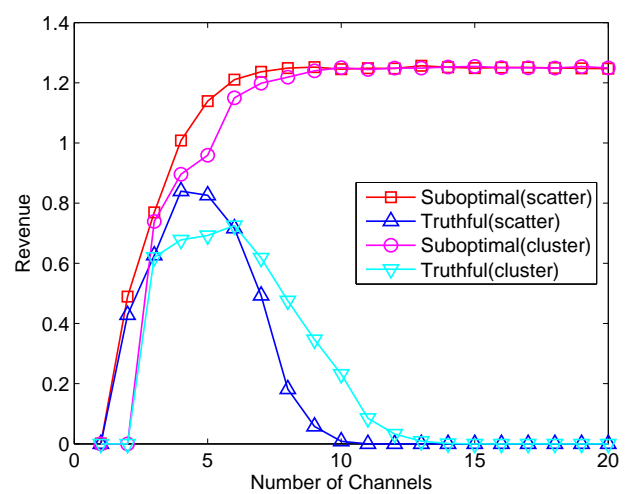

(a) Revenue

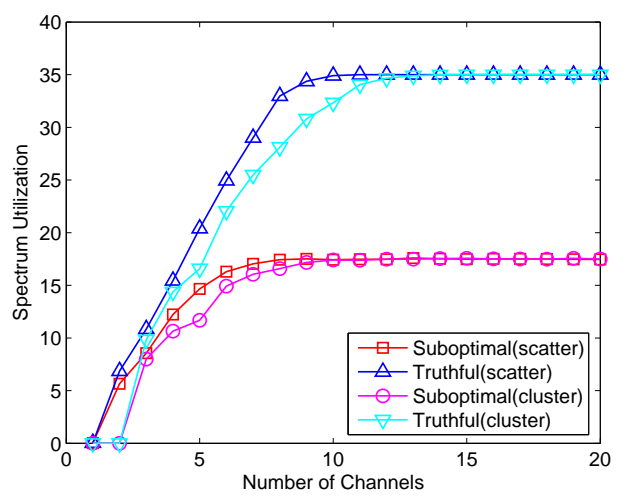

(b) Spectrum utilization

Figure 6: Comparison for scattered and clustered requests: (a) revenue (b) spectrum utilization utilization

within an alternative spectrum sharing model of interference temperature. Different from these works, our work focuses on revenue maximization for spectrum holders while considering both incentive-comparability and interference constraints.

There are also studies related to the operation of wireless service providers. [22] considers the problem of spectrum sharing among a primary user and multiple secondary users. They formulate the problem as an oligopoly market competition and use a Cournot game to obtain the spectrum allocation for secondary users. [8] studies the strategies of wireless service providers in future dynamic spectrum access systems, where they need to consider both spectrum acquisition competition and service provision competition. In this paper, the spatial reuse of spectrum is considered and auction is used as the market institution.

\section{CONCLUSION}

In this paper, we presented a general secondary spectrum trading framework, and in particular, an auction framework, for future spectrum holders and wireless service providers. Under such a framework a primary license holder can sell access to its unused or under-utilized spectrum resources in the form of certain fine-grained spectrum-space-time unit. We motivated such a framework and presented some of the design principles. How to maximize auction revenue while 
enforcing truthful bidding, and how to resolve spectrum interference are two key challenges we addressed in this paper. We proposed two auctions based on virtual valuation. One is optimal in revenue generation using the VCG mechanism, but it has high complexity. The other one uses a greedy like allocation and payment scheme. It has suboptimal revenue but is computationally much more efficient. These auctions provide primary users sufficient incentive to share their spectrum and thus make dynamic spectrum access more practical. The future work includes extension of the auction model and related analysis to the other spectrum request formats.

\section{ACKNOWLEDGEMENT}

The research was support in part by grants from RGC under the contracts CERG 622407, 622508 and N HKUST609/07, by grant from HKUST under the contract RPC06/07.EG05, the NSFC oversea Young Investigator grant under Grant No. 60629203, and National Basic Research Program of China (973 Program) under Grant No. 2006CB303100.

\section{REFERENCES}

[1] M.A. McHenry, P.A. Tenhula, D. McCloskey, D.A. Roberson, and C.S. Hood. Chicago spectrum occupancy measurements \& analysis and a long-term studies proposal. In Proceedings of the first international workshop on Technology and policy for accessing spectrum. ACM New York, NY, USA, 2006.

[2] R. Chandra, R. Mahajan, T. Moscibroda, R. Raghavendra, and P. Bahl. A case for adapting channel width in wireless networks. ACM Sigcomm 2008.

[3] C. Cordeiro, K. Challapali, and M. Ghosh. Cognitive PHY and MAC layers for dynamic spectrum access and sharing of TV bands. In Proceedings of the first international workshop on Technology and policy for accessing spectrum. ACM New York, NY, USA, 2006.

[4] I.F. Akyildiz, W.Y. Lee, M.C. Vuran, and S. Mohanty. NeXt generation/dynamic spectrum access/cognitive radio wireless networks: A survey. Computer Networks, 50(13):2127-2159, 2006.

[5] S. Haykin. Cognitive radio: brain-empowered wireless communications. IEEE Journal on Selected Areas in Communications, , 23(2):201-220, 2005.

[6] Q. Zhao, L. Tong, A. Swami, and Y. Chen. Decentralized cognitive MAC for opportunistic spectrum access in ad hoc networks: A POMDP framework. IEEE Journal on Selected Areas in Communications, 25(3):589-600, 2007.

[7] P. Cramton. The FCC Spectrum Auctions: An Early Assessment. Journal of Economics \& Management Strategy, 6(3):431-495, 1997.

[8] J. Jia and Q. Zhang. Competitions and dynamics of duopoly wireless service providers in dynamic spectrum market. In Proceedings of the 9th ACM international symposium on Mobile ad hoc networking and computing (ACM MobiHoc), pages 313-322. ACM New York, NY, USA, 2008.

[9] S. Huang, X. Liu, and Z. Ding. Opportunistic spectrum access in cognitive radio networks. In The 27th Conference on Computer Communications (IEEE INFOCOM), pages 1427-1435, 2008.
[10] DN Hatfield and PJ Weiser. Property rights in spectrum: taking the next step. In First IEEE International Symposium on New Frontiers in Dynamic Spectrum Access Networks (IEEE DySPAN), pages 43-55, 2005.

[11] L. Doyle and T. Forde. Towards a Fluid Spectrum Market for Exclusive Usage Rights. In 2nd IEEE International Symposium on New Frontiers in Dynamic Spectrum Access Networks (IEEE DySPAN), pages 620-632, 2007.

[12] R.B. Myerson. Optimal Auction Design. MATH. OPER. RES., 6(1):58-73, 1981.

[13] N. Nisan. Algorithmic Game Theory. Cambridge University Press, 2007.

[14] D. Lehmann, L.I. Oćallaghan, and Y. Shoham. Truth revelation in approximately efficient combinatorial auctions. Journal of the ACM, 49(5):577-602, 2002.

[15] X. Zhou, S. Gandhi, S. Suri, and H. Zheng. eBay in the Sky: strategy-proof wireless spectrum auctions. In Proceedings of the 14 th ACM international conference on Mobile computing and networking (ACM MobiCom), pages 2-13. ACM New York, NY, USA, 2008.

[16] A. Mu'alem and N. Nisan. Truthful approximation mechanisms for restricted combinatorial auctions. Games and Economic Behavior, 2008.

[17] V. Grimm, F. Riedel, and E. Wolfstetter. Low price equilibrium in multi-unit auctions: the GSM spectrum auction in Germany. International Journal of Industrial Organization, 21(10):1557-1569, 2003.

[18] T. Borgers, C. Dustmann, and G. Street. Strange Bids: Bidding Behaviour in the United Kingdom's Third Generation Spectrum Auction. Economic Journal, 115(505):551-578, 2005.

[19] Y. Wu, B. Wang, K.J.R. Liu, and T.C. Clancy. A Multi-Winner Cognitive Spectrum Auction Framework with Collusion-Resistant Mechanisms. In 3rd IEEE Symposium on New Frontiers in Dynamic Spectrum Access Networks (IEEE DySPAN), pages 1-9, 2008.

[20] A.P. Subramanian, M. Al-Ayyoub, H. Gupta, S.R. Das, and M.M. Buddhikot. Near-Optimal Dynamic Spectrum Allocation in Cellular Networks. In 3rd IEEE Symposium on New Frontiers in Dynamic Spectrum Access Networks (IEEE DySPAN), pages 1-11, 2008.

[21] J. Huang, R.A. Berry, and M.L. Honig. Auction-Based Spectrum Sharing. Mobile Networks and Applications, 11(3):405-408, 2006.

[22] D. Niyato and E. Hossain. A game-theoretic approach to competitive spectrum sharing in cognitive radio networks. In IEEE Wireless Communications and Networking Conference (IEEE WCNC), pages 16-20, 2007. 